\title{
Effect of Phytosomal Curcumin on Circulating Levels of Adiponectin and Leptin in Patients with Non-Alcoholic Fatty Liver Disease: A Randomized, Double-Blind, Placebo-Controlled Clinical Trial
}

\author{
Seyed Reza Mirhafez ${ }^{1,2}$, Azam Rezaei Farimani ${ }^{1,2}$, Maryam Dehhabe ${ }^{2}$, Mohammad Bidkhori ${ }^{3}$, Mitra Hariri ${ }^{1}$, Bibi \\ Fatemeh Nobakht Motlagh Ghouchani ${ }^{1}$, Fatemeh Abdollahi ${ }^{3}$
}

1) Noncommunicable Diseases Research Center, Neyshabur University of Medical Sciences, Neyshabur

2) Department of Basic Medical Sciences, Neyshabur University of Medical Sciences, Neyshabur

3) Department of Public Health, Neyshabur University of Medical Sciences, Neyshabur, Iran

\author{
Address for correspondence: \\ Azam Rezaei Farimani \\ Noncommunicable Diseases \\ Research Center, Neyshabur \\ University of Medical Sciences, \\ Neyshabur, Iran. \\ azam_rezaei1@yahoo.com
}

\begin{abstract}
Background \& Aims: Non-alcoholic fatty liver disease (NAFLD) is associated with insulin resistance and changes in serum adipocytokine levels. The aim of the current study was to evaluate the effect of phytosomal curcumin on serum adiponectin and leptin levels in patients with NAFLD.

Methods: In this randomized double-blind, placebo-controlled trial, 65 eligible patients were randomly allocated into curcumin and placebo recipient groups using a blocked randomized technique. Parameters of weight, height, body mass index (BMI), fasting blood sugar (FBS), lipid profile, aspartate aminotransferase (AST), alanine aminotransferase (ALT), adiponectin, leptin, and the leptin:adiponectin ratio were measured at baseline and eight weeks after intervention.

Results: High-density lipoprotein cholesterol (HDL-C) levels increased significantly in the curcumin group compared to the placebo group $(\mathrm{p}=0.01)$. Serum adiponectin levels increased significantly $(\mathrm{p}<0.001)$ and serum leptin levels decreased significantly $(\mathrm{p}<0.001)$ with a decrease in the leptin: adiponectin ratio in the curcumin group compared to the placebo group after 8 weeks of intervention.

Conclusions: Non-alcoholic fatty liver disease was associated with changes in serum adipokines levels. Phytosomal curcumin effectively improved leptin and adiponectin levels. It is possible that curcumin efficacy will increase with long-term use of higher doses of this substance.
\end{abstract}

Key words: non-alcoholic fatty liver disease - curcumin - adiponectin - leptin

Abbreviations: ALT: alanine aminotransferase; AST: aspartate aminotransferases; BMI: body mass index; FBS: fasting blood sugar; HDL-C: high-density lipoprotein cholesterol; LDL-C: low-density lipoprotein cholesterol; NAFLD: non-alcoholic fatty liver disease; VLDL: very low density lipoprotein; TG: triglycerides.

\section{INTRODUCTION}

Non-alcoholic fatty liver disease (NAFLD) is a common liver disease that threatens the health of adults and children globally, with the highest prevalence being in the Middle East and South America [1]. This pathology is diagnosed by evidence of hepatic steatosis (accumulation of free fatty acids and triglycerides) and the absence of secondary causes of liver fat accumulation. Steatosis without necro-inflammatory injury and steatosis with inflammatory injury of hepatocytes are considered to be types of NAFLD.
Increased lipid synthesis, excess fatty acid delivery, reduced lipid export in the form of very low density lipoprotein (VLDL), reduced lipid oxidation or imbalances in any of these pathways can be a cause of lipid accumulation in the liver [2]. Nearly $90 \%$ of NAFLD cases have at least one specific metabolic syndrome characteristic [3]. Obesity, type 2 diabetes, high blood pressure, and dyslipidemia are components of the metabolic syndrome that are strongly associated with NAFLD.

In case of insulin resistance, increased lipolysis and increased release and flux of fatty acids to the liver are associated with excessive synthesis and fat accumulation in hepatocytes [4]. This event is the first to affect the liver, causing it to become susceptible to secondary hits from oxidative stress, inflammatory cytokines and adipocytokines [5]. Evidence suggests that adipocytokines are involved in NAFLD pathology by affecting insulin resistance [6]. Adipose tissue acts as an energy source in the form of lipids and also produces and secretes the pro- and anti-inflammatory proteins called adipokines (adipocytokines). 
Leptin and adiponectin are important adipokines that affect insulin sensitivity. These proteins are produced mainly by adipocytes [7]. Changes in fat mass increase the circulating levels of leptin and decrease the levels of adiponectin in obese individuals [8]. Considering the relationship between adipose tissue and the liver, disturbances in adipokines production can affect the liver metabolism and hepatic insulin sensitivity [9].

Adiponectin is a $30 \mathrm{kDa}$ protein that prevents hepatic fat accumulation by decreasing de novo lipogenesis and increasing liver sensitivity to insulin. The liver expresses adiponectin receptors AdipoR1 and AdipoR2 as the main targets of adiponectin. Adiponectin is an anti-inflammatory agent that reduces plasma and liver tumor necrosis factor a (TNF- $\alpha$ ) concentrations and increases production of interleukin 10 (IL-10). Leptin is a $16 \mathrm{kDa}$ protein that regulates the energy balance by acting on receptors in the arcuate nucleus of the hypothalamus. Decreased sensitivity to leptin occurs in obesity and insulin resistance $[10,11]$. High levels of leptin have been reported in patients with NAFLD and are an indicator of the severity of the disease [12].

At present, there is no definitive cure for NAFLD. Current treatments are the use of insulin sensitizers, weight loss, diabetes control and exercise. As a result, understanding the new therapeutic approaches will require extensive research. Lifestyle, physical activity, and dietary composition are among the factors that can influence NAFLD. Today, the use of herbal medicine and bioactive natural products have been considered $[13,14]$.

A body of evidence has shown that curcumin has a positive impact on NAFLD $[15,16]$. Curcumin $[1,7$-bis-(4hydroxy-3-methoxyphenyl)-1, 6-heptadiene-3, 5-dione] is an active phytochemical component of turmeric. The turmeric plant (Curcuma longa Linn) is used to create a dietary spice that is common to most parts of Asia and offers multiple biological and pharmacological effects. The anti-inflammatory, antioxidant, anti-atherosclerotic, cardioprotective, and anti-cancer properties of curcumin have been investigated [17]. Curcumin has been reported to reduce the synthesis of pro-inflammatory cytokines and increase anti-inflammatory cytokines through different biological mechanisms. In this study, we aimed to evaluate the effect of curcumin on serum levels of adiponectin, leptin and the leptin:adiponectin ratio in patients with NAFLD.

\section{METHODS}

\section{Study design}

A randomized, double-blind, placebo-controlled, clinical trial was designed to investigate the effect of phytosomal curcumin on serum adiponectin and leptin levels for eight weeks of treatment. The volunteers included 74 patients with a previous history of NAFLD or new cases referred to the gastroenterology clinic of 22 Bahman Hospital in the city of Neyshabur, Iran. The inclusion criteria for participation in the study were age over 18 years of either gender, confirmed diagnosis of NAFLD (bright liver findings) from liver ultrasound scans. Exclusion criteria were a diagnosis of autoimmune or viral hepatitis, Wilson's disease, alcohol consumption, pregnancy, diabetes, thyroid disorders, malignancy, lactation, hemochromatosis, and use of dietary supplements and other sources of increased liver enzyme levels.

Eligible individuals completed the informed consent form. They were free to leave the study at any time. The study designer used the blocked randomized technique to allocate participants to either the curcumin (A) or placebo (B) recipient groups with a block size of four for the two groups (ABAB, BAAB, $\mathrm{BABA}$, etc.). Only the main researcher knew the contents of the packages and the types of drugs. Phospholipidated curcumin (Meriva; each capsule composed of $250 \mathrm{mg}$ /day equivalent to $50 \mathrm{mg} /$ day pure curcumin) and placebo capsules with the same appearance were given to patients in A and B bottles for eight weeks.

\section{Blood sampling and measurements}

Anthropometric parameters of weight, height, and body mass index (BMI) were evaluated at baseline and at the end of intervention using an InBody 770 connected to a BSM 370. In the fasting state, $10 \mathrm{~mL}$ of venous blood was collected from each patient at baseline and eight weeks later. The blood samples were centrifuged for $10 \mathrm{~min}$ at $2500 \mathrm{rpm}$ to separate the serum and stored at $-80^{\circ} \mathrm{C}$. Laboratory tests for liver enzymes (ALT and AST), fasting blood glucose (FBS), and lipid panel (total cholesterol, high-density lipoprotein cholesterol (HDL-C), low-density lipoprotein cholesterol (LDL-C) and triglycerides (TG) were measured with an autoanalyzer (BT-2000) (Pars Azmoun; Iran). Enzyme-linked immune sorbent assay (ELISA) based on sandwich-assay using two specific and highly affine antibodies was used to assay the serum adiponectin and leptin (Mediagnost; Germany).

\section{Statistical analysis}

Version 20 of SPSS Statistics (SPSS; USA) was used for statistical analysis and data management. The mean, standard deviation, frequency and percentage were reported to describe the data. The paired sample $t$-test was used to determine the mean difference between two related groups before and after treatment (within-group comparison). The independent sample $t$-test was used for between-group comparisons. In the general linear model, univariate analysis was carried out to eliminate the effect of confounding variables. A p-value $<0.05$ was considered statistically significant. The protocol of this trial was registered in Iranian Registry of Clinical Trials center, IRCTID: IRCT2015052322381N1.

\section{Ethic and informed consent}

This project was approved by the Research Ethics Committee of the Neyshabur University of Medical Sciences (IR.NUMS.REC.1395.48). Informed consent was obtained from all participants.

\section{RESULTS}

Sixty-five patients with NAFLD who met the inclusion criteria were randomly assigned to either the curcumin $(n=33)$ or placebo $(n=32)$ groups. A total of 61 patients completed the study. Three patients in the placebo group and one in the curcumin group (Fig. 1) were lost to follow-up. There were no significant differences between groups at baseline for the clinical 


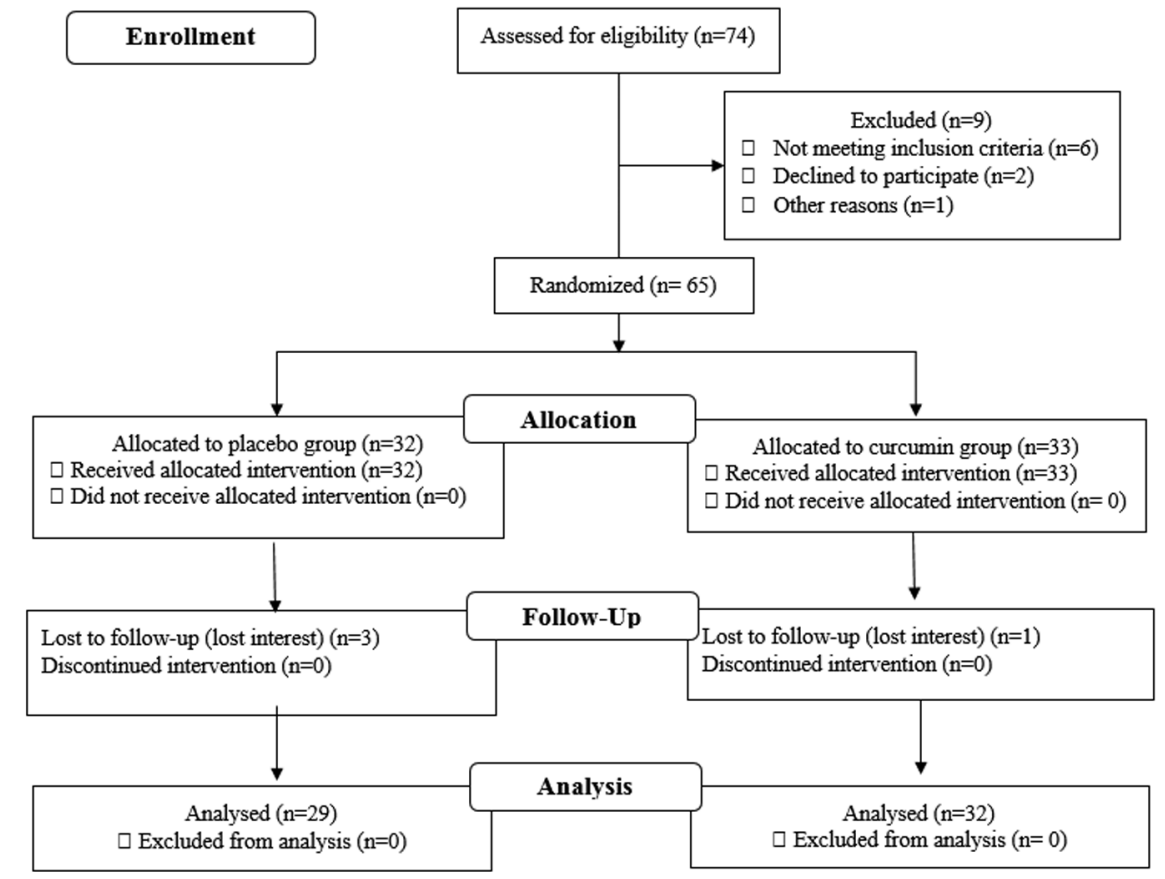

Fig. 1. Flowchart of patients entered into the study.

and demographic characteristics. However, serum levels of total cholesterol $(p=0.02)$, and LDL-C $(p=0.01)$ were higher in the curcumin group that in the placebo group (Table I).

\section{Effect of curcumin on weight and BMI}

The anthropometric parameters of weight and BMI were compared at baseline and eight weeks after intervention in both groups. Within-group comparisons showed that weight $(\mathrm{p}=0.13)$ and BMI $(\mathrm{p}=0.12)$ increased in the placebo group and decreased in curcumin-treated group (weight; $\mathrm{p}=0.16$ and BMI; $\mathrm{p}=0.12$ ), although the changes were not significant (Table II). No statistically significant changes were observed in between-group analysis for weight $(\mathrm{p}=0.07)$ and $\mathrm{BMI}(\mathrm{p}=0.08)$ (Table III).

Table I. Baseline demographic and clinical characteristics of study groups.

\begin{tabular}{lccc}
\hline Variables & Curcumin & Placebo & $\mathrm{p}$ \\
\hline Age (years) & $44.8(11.14)$ & $40.7(11.83)$ & 0.12 \\
Gender & & & \\
Male $(\%)$ & $18(48.6)$ & $19(51.4)$ & \\
Female (\%) & $14(63.6)$ & $8(36.4)$ & 0.21 \\
Weight $(\mathrm{kg})$ & $83.86(20.81)$ & $76.99(16.68)$ & 0.21 \\
BMI $\left(\mathrm{kg} / \mathrm{m}^{2}\right)$ & $30.06(5.76)$ & $27.72(5.97)$ & 0.19 \\
Leptin $(\mathrm{ng} / \mathrm{mL})$ & $23.21(16.93)$ & $15.01(18.92)$ & 0.08 \\
Adiponectin $(\mathrm{ng} / \mathrm{mL})$ & $14.35(7.72)$ & $11.85(6.75)$ & 0.21 \\
Leptin: adiponectin & $1.81(1.90)$ & $1.59(3.22)$ & 0.72 \\
FBS (mg/dL) & $109.83(49.68)$ & $105.92(21.56)$ & 0.69 \\
TG (mg/dL) & $130.46(87.27)$ & $139.19(58.51)$ & 0.66 \\
Total cholesterol (mg/dL) & $204.21(37.68)$ & $183.64(29.14)$ & 0.02 \\
HDL-C (mg/dL) & $41.43(13.86)$ & $45.00(10.55)$ & 0.18 \\
LDL-C (mg/dL) & $138.40(40.39)$ & $108.71(24.14)$ & 0.001 \\
AST (mg/dL) & $32.45(18.60)$ & $25.18(11.11)$ & 0.08 \\
ALT (mg/dL) & $47.66(35.20)$ & $40.07(19.82)$ & 0.32 \\
\hline
\end{tabular}

Results are expressed in terms of mean (SD). p-values refer to comparison between curcumin and placebo groups at the baseline. ALT: alanine aminotransferase; AST: aspartate aminotransferase; FBS: fasting blood sugar; HDL-c: high-density lipoprotein cholesterol; LDL-c: low-density lipoprotein cholesterol; TG: triglyceride. 
Table II. Within-group comparisons of parameters between curcumin and placebo groups

\begin{tabular}{|c|c|c|c|c|c|c|c|c|}
\hline \multirow[b]{2}{*}{ Variables } & \multicolumn{4}{|c|}{ Curcumin } & \multicolumn{4}{|c|}{ Placebo } \\
\hline & Before & After & $95 \% \mathrm{CI}$ & $\mathrm{p}$ & Before & After & $95 \% \mathrm{CI}$ & $\mathrm{p}$ \\
\hline Weight $(\mathrm{kg})$ & $83.86(20.81)$ & $83.09(20.90)$ & $-0.33-1.86$ & 0.16 & $77.99(16.68)$ & $79.99(11.60)$ & $-7.00-1.00$ & 0.13 \\
\hline BMI $\left(\mathrm{kg} / \mathrm{m}^{2}\right)$ & $30.06(5.76)$ & $29.75(5.84)$ & $0.08-0.68$ & 0.12 & $27.72(5.97)$ & $28.78(4.22)$ & $-2.48-0.35$ & 0.13 \\
\hline Leptin $(\mathrm{ng} / \mathrm{mL})$ & $23.21(16.93)$ & $17.85(14.11)$ & $1.66-9.06$ & $<0.001$ & $15.01(18.92)$ & $15.86(17.06)$ & $-4.36-2.64$ & 0.61 \\
\hline Adiponectin (ng/mL) & $14.35(7.72)$ & $18.23(9.75)$ & $-5.80--1.94$ & $<0.001$ & $11.85(6.75)$ & $10.87(4.95)$ & $-0.29-2.25$ & 0.12 \\
\hline Leptin: adiponectin & $1.81(1.90)$ & $1.12(1.09)$ & $0.30-1.08$ & $<0.001$ & $1.59(3.22)$ & $1.97(4.07)$ & $-0.90-0.12$ & 0.13 \\
\hline $\mathrm{FBS}(\mathrm{mg} / \mathrm{dL})$ & $109.83(49.68)$ & $101.00(8.02)$ & $-8.91-26.59$ & 0.31 & $105.92(21.56)$ & $107.74(22.69)$ & $-6.03-2.40$ & 0.38 \\
\hline $\mathrm{TG}(\mathrm{mg} / \mathrm{dL})$ & $130.46(87.27)$ & $130.37(83.68)$ & $-14.95-15.09$ & 0.99 & $139.19(58.51)$ & $140.53(66.39)$ & $-17.08-14.39$ & 0.86 \\
\hline Total cholesterol (mg/dL) & $204.21(37.68)$ & $195.12(40.61)$ & $-0.92-19.11$ & 0.07 & $183.64(29.14)$ & $187.76(31.34)$ & $-6.88-15.12$ & 0.44 \\
\hline HDL-C (mg/dL) & $41.43(13.86)$ & $41.93(13.93)$ & $-3.35-2.36$ & 0.72 & $45.00(10.55)$ & $42.41(10.59)$ & $-0.50-5.22$ & 0.05 \\
\hline LDL-C (mg/dL) & $138.40(40.39)$ & $130.98(42.75)$ & $(-3.00-17.83)$ & 0.15 & $108.71(24.14)$ & $119.73(24.60)$ & $-19.83--2.20$ & 0.01 \\
\hline $\operatorname{AST}(\mathrm{mg} / \mathrm{dL})$ & $32.45(18.60)$ & $30.45(15.61)$ & $(-3.08-7.08)$ & 0.42 & $25.18(11.11)$ & $27.62(9.89)$ & $(-7.07-2.18)$ & 0.28 \\
\hline $\operatorname{ALT}(\mathrm{mg} / \mathrm{dL})$ & $47.66(35.20)$ & $41.54(22.98)$ & $(-5.44-15.57)$ & 0.33 & $40.07(19.82)$ & $41.29(19.29)$ & $(-7.35-5.09)$ & 0.69 \\
\hline
\end{tabular}

Results are expressed in terms of mean (SD). p-value: Within-group comparisons at the baseline and 8 weeks after intervention. (For abbreviations see Table I)..

Table III. Between-group comparison of parameters between curcumin and placebo groups

\begin{tabular}{lccc}
\hline Variables & Curcumin & Placebo & $\mathrm{p}$ \\
\hline Weight $(\mathrm{kg})$ & $-0.76(2.78)$ & $2.00(8.79)$ & 0.07 \\
BMI $\left(\mathrm{kg} / \mathrm{m}^{2}\right)$ & $1.06(3.27)$ & $-0.31(0.96)$ & 0.08 \\
Leptin $(\mathrm{ng} / \mathrm{mL})$ & $-5.36(10.26)$ & $0.85(8.86)$ & $<0.001$ \\
Adiponectin $(\mathrm{ng} / \mathrm{mL})$ & $3.87(5.17)$ & $-0.97(5.17)$ & $<0.001$ \\
Leptin: adiponectin & $-0.69(1.05)$ & $0.38(1.18)$ & $<0.001$ \\
FBS $(\mathrm{mg} / \mathrm{dL})$ & $-8.83(48.41)$ & $1.81(10.66)$ & 0.24 \\
TG $(\mathrm{mg} / \mathrm{dL})$ & $-0.09(41.60)$ & $1.34(38.97)$ & 0.89 \\
Total cholesterol $(\mathrm{mg} / \mathrm{dL})$ & $-9.09(27.79)$ & $4.12(26.65)$ & 0.08 \\
HDL-C (mg/dL) & $0.49(7.93)$ & $-3.98(5.76)$ & 0.01 \\
LDL-C $(\mathrm{mg} / \mathrm{dL})$ & $-7.41(27.91)$ & $11.02(20.38)$ & 0.10 \\
AST $(\mathrm{mg} / \mathrm{dL})$ & $-2.00(14.33)$ & $2.44(11.69)$ & 0.19 \\
ALT $(\mathrm{mg} / \mathrm{dL})$ & $-6.12(26.60)$ & $1.22(15.96)$ & 0.17 \\
\hline
\end{tabular}

p-value: Between-group comparisons after intervention. (For abbreviations see Table I).

\section{Effect of curcumin on serum levels of FBS and lipids}

Within-group comparisons revealed that serum FBS levels increased in the placebo group $(\mathrm{p}=0.38)$ and decreased in the curcumin group ( $\mathrm{p}=0.31)$, although the changes were not statistically significant (Table II). There was no significant change in comparison of means between groups $(\mathrm{p}=0.24)$ (Table III).

The serum levels of total cholesterol and TG increased non-significantly in the placebo group $(\mathrm{p}=0.44$ and $\mathrm{p}=0.86$, respectively) and non-significantly decreased in the curcumin group ( $p=0.07$ and $p=0.99$, respectively). There was a significant increase in serum LDL-C $(\mathrm{p}=0.01)$ and a borderline decrease in HDL-C $(p=0.05)$ in the placebo group. In the curcumin group, there was a decrease in LDL- $C(p=0.15)$ and an increase in HDL-C $(\mathrm{p}=0.72)$, but the changes were not statistically significant (Table II). In between-group analysis, significant differences were observed in serum LDL-C $(p=0.01)$ and HDL-C $(p=0.01)$ in the curcumin group compared to the placebo group (Table III), although the change in serum LDL-C was not significant after adjusting for baseline differences between groups $(\mathrm{p}=0.10)$.

\section{Effect of curcumin on serum levels of liver enzymes}

Within-group comparison showed that serum levels of liver enzymes were not significantly elevated in the placebo group (ALT: $\mathrm{p}=0.96$; AST: $\mathrm{p}=0.28$ ) and non-significantly decreased in the curcumin group (ALT: $p=0.33$; AST: $p=0.42$ ) (Table II). Comparison of the change values showed no significant differences between groups (ALT: $p=0.17$; AST: $p=0.19$ ) (Table III).

Effect of curcumin on serum levels of leptin and adiponectin hormones

The mean serum levels of leptin and adiponectin were compared at baseline and eight weeks after intervention in the placebo and the curcumin groups. Within-group analysis showed no significant difference in serum levels of leptin $(\mathrm{p}=0.61)$ and adiponectin $(\mathrm{p}=0.12)$ in the placebo group. By contrast, there was a significant decrease in serum leptin $(p<0.001)$ and a significant increase in serum adiponectin $(p<0.001)$ in the curcumin group (Table II). Analysis of between-group changes revealed a significant decrease in serum leptin $(\mathrm{p}<0.001)$ and a significant increase in serum adiponectin $(\mathrm{p}<0.001)$ in the curcumin group compared to the placebo group. The leptin: adiponectin ratio also significantly decreased in the curcumin group compared to the placebo group $(\mathrm{p}<0.001)$ (Table III). These changes remained statistically significant after adjustment for baseline differences between groups.

\section{Bivariate correlations}

Bivariate correlation analysis in the curcumin group showed that changes in serum levels of adiponectin correlated with changes in serum levels of ALT $(p=0.01)$. Changes in the serum leptin levels correlated with changes in the serum levels of LDL-C $(\mathrm{p}=0.01)$, and total cholesterol $(\mathrm{p}=0.03)$. The changes in the leptin: adiponectin ratio correlated with changes in serum levels of total cholesterol $(\mathrm{p}=0.01)$. No significant correlations were observed in the placebo group (Table IV). 
Table IV. Bivariate correlations between parameters in the curcumin of placebo groups

\begin{tabular}{|c|c|c|c|c|c|c|c|c|c|c|c|c|}
\hline \multirow[t]{3}{*}{ Variables } & \multicolumn{6}{|c|}{ Curcumin } & \multicolumn{6}{|c|}{ Placebo } \\
\hline & \multicolumn{2}{|c|}{ Leptin } & \multicolumn{2}{|c|}{ Adiponectin } & \multicolumn{2}{|c|}{ Leptin: adiponectin } & \multicolumn{2}{|c|}{ Leptin } & \multicolumn{2}{|c|}{ Adiponectin } & \multicolumn{2}{|c|}{ Leptin: adiponectin } \\
\hline & $\mathrm{r}$ & $\mathrm{p}$ & $\mathrm{r}$ & $\mathrm{p}$ & $\mathrm{r}$ & $\mathrm{p}$ & $\mathrm{r}$ & $\mathrm{p}$ & $\mathrm{r}$ & $\mathrm{p}$ & $\mathrm{r}$ & $\mathrm{p}$ \\
\hline Weight & 0.15 & 0.43 & -0.31 & 0.13 & -0.16 & 0.43 & 0.10 & 0.66 & 0.27 & 0.24 & -0.13 & 0.59 \\
\hline BMI & 0.14 & 0.47 & -0.35 & 0.08 & -0.16 & 0.44 & 0.09 & 0.68 & 0.27 & 0.26 & -0.12 & 0.58 \\
\hline FBS & 0.16 & 0.39 & -0.02 & 0.91 & 0.18 & 0.33 & -0.27 & 0.17 & 0.15 & 0.45 & -0.05 & 0.82 \\
\hline LDL-C & 0.45 & 0.01 & 0.15 & 0.43 & 0.33 & 0.08 & -0.33 & 0.12 & 0.04 & 0.08 & -0.04 & 0.86 \\
\hline HDL-C & 0.17 & 0.35 & 0.19 & 0.30 & 0.09 & 0.63 & 0.16 & 0.42 & -0.14 & 0.52 & 0.09 & 0.66 \\
\hline Total cholesterol & 0.40 & 0.03 & 0.13 & 0.47 & 0.49 & 0.01 & -0.29 & 0.15 & -0.30 & 0.17 & 0.08 & 0.72 \\
\hline TG & 0.02 & 0.88 & 0.12 & 0.51 & 0.30 & 0.10 & 0.01 & 0.95 & 0.20 & 0.34 & 0.06 & 0.78 \\
\hline AST & 0.07 & 0.67 & -0.32 & 0.08 & 0.01 & 0.97 & 0.03 & 0.86 & -0.01 & 0.99 & 0.13 & 0.95 \\
\hline ALT & 0.06 & 0.72 & -0.46 & 0.01 & 0.01 & 0.95 & 0.05 & 0.78 & -0.22 & 0.29 & 0.08 & 0.69 \\
\hline
\end{tabular}

Results of Pearson's Correlation. (For abbreviations see Table I).

\section{Safety}

The curcumin was found to be safe and no patients reported side effects with its use.

\section{DISCUSSION}

A curcumin-phosphatidylcholine phytosomal complex with high bioavailability [18] was used to evaluate the effect of curcumin on the serum levels of adiponectin, leptin and the leptin:adiponectin ratio in patients with NAFLD. It is known that obesity is associated with an increase in inflammatory factors, insulin resistance and NAFLD development [19]. The results of a meta-analysis study of 21 cohort studies indicated that there is a relationship between BMI and NAFLD, with a 3.5-fold higher risk of disease for obese subjects than normalweight subjects [20]. According to the evidence [20,21], the majority of patients in this study were overweight or obese.

The findings of this trial indicated that curcumin did not significantly reduce weight and BMI in the curcumin group compared to the placebo group. Some studies have shown that intake of curcumin significantly decreased the weight and BMI of overweight subjects with metabolic syndrome [22]. In this study, it is possible that the effect of curcumin on anthropometric parameters increased with an increase in the dose or duration of intervention in the curcumin-treated group. Curcumin can be effective for weight loss by activating AMP-activated protein kinase (AMPK) and regulating lipid metabolism [23]. AMPK by phosphorylation of acetyl-CoA carboxylase 1 (ACC1) and sterol regulatory element-binding protein 1c (SREBP1c) inhibits synthesis of fatty acids, cholesterol, and triglycerides and stimulates fatty acid uptake and $\beta$-oxidation [24].

Curcumin can also increase $\beta$-oxidation of fatty acids by increasing expression of carnitine palmitoyltransferase1 (CPT-1). It has been proven that curcumin can increase the rate of energy expenditure in high-fat-fed mice [25]. It also has been shown that curcumin decreases plasma glucose levels by activation of glycolytic enzymes, inhibition of gluconeogenic enzymes and improving insulin sensitivity [26]. Consistent with these findings, curcumin reduced serum levels of FBS in the curcumin-treated group compared to the placebo group in the current study.
Curcumin could also be effective in reducing serum lipid levels, so that serum levels of TG, LDL-C and total cholesterol were not significantly decreased in the curcumin group compared with the placebo group. Studies have shown that curcumin can decrease the lipid profile by stimulating the peroxisome proliferator-activated receptor (PPAR $\gamma)$ [27]. Panahi et al. reported that administration of curcumin (1000 $\mathrm{mg} /$ day for 8 weeks) significantly reduced the serum levels of total cholesterol, LDL-C, and triglycerides in patients with NAFLD [15].

Data from a systematic review and meta-analysis of randomized controlled trials revealed that curcumin had no effect on lipid parameters; however, heterogeneity in the study populations, duration of intervention, dose and the bioavailability of curcumin could affect outcomes [28]. Several studies have shown that curcumin enhances the expression of lipolytic genes (CPT- 1 and PPAR- $\gamma$ ) and reduces the expression of lipogenic genes (SREBP-1c, FAS and ACC) [25, 29]. The findings of the current study showed that serum levels of liver enzymes decreased in the curcumin group and increased in the placebo group; however, these differences were not statistically significant. It is possible that an increase in the duration of intervention or the use of higher doses of curcumin could be more effective.

A systematic review of randomized controlled trials suggested that high doses of curcumin supplementation $(\geq 1000$ $\mathrm{mg}$ /day) significantly reduced serum levels of ALT and AST in patients with NAFLD [30]. Several reports have shown that increased hepatic diacylglycerol content, along with activation of protein kinase $\mathrm{C}$ and inhibiting insulin signaling could induce insulin resistance in the NAFLD [31]. The activation of nuclear factor $\mathrm{kB}(\mathrm{NF}-\mathrm{kB})$ increased inflammatory cytokine production. It is known that altered adipokines levels are one pathological aspect of insulin resistance in NAFLD.

Curcumin can improve insulin sensitivity by inactivating NF-kB and reducing the expression of pro-inflammatory cytokines such as TNF- $\alpha[32,33]$. An increase in inflammatory cytokine levels, including IL-6 and TNF- $\alpha$, is associated with inhibition of adiponectin expression, leptin resistance and high leptin levels in obese NAFLD patients; therefore, impaired production and secretion of adipokines appear to occur in NAFLD [32]. Vayghan et al. [34] reported serum levels of leptin and adiponectin of 8.3 and $30.63 \mathrm{ng} / \mathrm{ml}$, respectively, in the 
healthy adults from Iran. In our study, changes in serum leptin and adiponectin levels were observed in patients with NAFLD and were associated with an increase in serum leptin levels and a decrease in serum adiponectin levels. The findings of the current study showed that curcumin effectively reduced the leptin levels and increased adiponectin levels in the curcumintreated group compared to the placebo group. Navekar et al. [35] showed that turmeric supplementation caused a significant reduction in the leptin levels of NAFLD patients.

There is limited evidence regarding the effects of curcumin treatment on adipokines in NAFLD. In an animal model study, it was found that curcumin increased adiponectin mRNA expression by decreasing the methylation of adiponectin DNA [36]. Chuengsamarn et al. [37] showed that curcumin increased adiponectin levels in type 2 diabetes. Another study on metabolic syndrome showed that curcumin significantly increased adiponectin levels and decreased leptin levels [38]. Curcumin increases adiponectin levels by downregulation of inflammation-related genes (NF- $\kappa$ B, IL-6, and TNF- $\alpha$ ) [29]. Moreover, curcumin reduces macrophage infiltration of white adipose tissue, increases adiponectin production and suppresses hepatic inflammatory factors [39].

The current study was limited by the lack of classification of patients according to the severity and degree of NAFLD, the small sample size, short-term intervention and consumption of a single dose of curcumin, although the curcumin used had a high bioavailability formulation. However, due to limited studies in this field, our study results could be effective, but more extensive research is required. It is recommended to investigate the effects of curcumin on adiponectin, leptin, and other adipocytokines in accordance with the severity of NAFLD and, in order to reduce nutritional intervention the patients should adhere to a controlled diet.

\section{CONCLUSION}

The most important finding of this study was the significant increase in serum adiponectin levels and a significant decrease in serum leptin levels after consumption of phytosomal curcumin by patients with NAFLD. It is possible that the effect of curcumin on other factors could increase with long-term use of higher doses of curcumin.

Conflicts of interest: Nothing to declare.

Authors' contributions: A.R.F. and S.R.M. designed this study. M.D. and A.R.F. performed the experiments. A.R.F. wrote the manuscript. M.B. analyzed the results. M.H., F.N.M and F.A. contributed to the design and implementation of the research.

Acknowledgement: The authors would like to show their gratitude to the Research Deputy of Neyshabur University of Medical Sciences for providing financial support and equipment [Grant number 198, 2017].

\section{REFERENCES}

1. Younossi Z, Anstee QM, Marietti M, et al. Global burden of NAFLD and NASH: trends, predictions, risk factors and prevention.
Nat Rev Gastroenterol Hepatol 2018;15:11-20. doi:10.1038/ nrgastro.2017.109

2. Brunt EM. Pathology of nonalcoholic fatty liver disease. Nat Rev Gastroenterol Hepatol 2010;7:195-203. doi:10.1038/ nrgastro.2010.21

3. Marchesini G, Bugianesi E, Forlani G, et al. Nonalcoholic fatty liver, steatohepatitis, and the metabolic syndrome. Hepatology 2003;37:917923. doi:10.1053/jhep.2003.50161

4. Lewis GF, Carpentier A, Adeli K, Giacca A. Disordered fat storage and mobilization in the pathogenesis of insulin resistance and type 2 diabetes. Endocr Rev 2002;23:201-229. doi:10.1210/edrv.23.2.0461

5. Marrero JA, Fontana RJ, Su GL, Conjeevaram HS, Emick DM, Lok AS. NAFLD may be a common underlying liver disease in patients with hepatocellular carcinoma in the United States. Hepatology 2002;36:1349-1354. doi:10.1053/jhep.2002.36939

6. Polyzos SA, Kountouras J, Zavos C. Nonalcoholic fatty liver disease: the pathogenetic roles of insulin resistance and adipocytokines. Curr Mol Med 2009;9:299-314. doi:10.2174/156652409787847191

7. Yadav A, Kataria MA, Saini V, Yadav A. Role of leptin and adiponectin in insulin resistance. Clin Chim Acta 2013;417:80-84. doi:10.1016/j. cca.2012.12.007

8. Stern JH, Rutkowski JM, Scherer PE. Adiponectin, leptin, and fatty acids in the maintenance of metabolic homeostasis through adipose tissue crosstalk. Cell Metab 2016;23:770-784. doi:10.1016/j.cmet.2016.04.011

9. Nawrocki AR, Rajala MW, Tomas E, et al. Mice lacking adiponectin show decreased hepatic insulin sensitivity and reduced responsiveness to peroxisome proliferator-activated receptor $\gamma$ agonists. J Biol Chem 2006;281:2654-2660. doi:10.1074/jbc.M505311200

10. Brennan AM, Mantzoros CS. Drug Insight: the role of leptin in human physiology and pathophysiology-emerging clinical applications. Nat Clin Pract Endocrinol Metab 2006;2:318-327. doi:10.1038/ ncpendmet0196

11. Pan H, Guo J, Su Z. Advances in understanding the interrelations between leptin resistance and obesity. Physiol Behav 2014;130:157-169. doi:10.1016/j.physbeh.2014.04.003

12. Polyzos SA, Aronis KN, Kountouras J, Raptis DD, Vasiloglou MF Mantzoros CS. Circulating leptin in non-alcoholic fatty liver disease: a systematic review and meta-analysis. Diabetologia 2016;59:30-43. doi:10.1007/s00125-015-3769-3

13. Baghernya M, Nobili V, Blesso CN, Sahebkar A. Medicinal plants and bioactive natural compounds in the treatment of non-alcoholic fatty liver disease: a clinical review. Pharmacol Res 2018;130:213-240. doi:10.1016/j.phrs.2017.12.020

14. Farimani AR, Hariri M, Azimi-Nezhad M, Borji A, Zarei S, Hooshmand E. The effect of n-3 PUFAs on circulating adiponectin and leptin in patients with type 2 diabetes mellitus: a systematic review and metaanalysis of randomized controlled trials. Acta Diabetol 2018;55:641-652. doi:10.1007/s00592-018-1110-6

15. Panahi Y, Kianpour P, Mohtashami R, Jafari R, Simental-Mendía LE, Sahebkar A. Curcumin lowers serum lipids and uric acid in subjects with nonalcoholic fatty liver disease: a randomized controlled trial. J Cardiovasc Pharmacol 2016;68:223-229. doi:10.1097/ FJC.0000000000000406

16. Panahi Y, Kianpour P, Mohtashami R, Jafari R, Simental-Mendía LE, Sahebkar A. Efficacy and safety of phytosomal curcumin in nonalcoholic fatty liver disease: a randomized controlled trial. Drug Res 2017;67:244-251. doi:10.1055/s-0043-100019

17. Hewlings SJ, Kalman DS. Curcumin: A Review of Its' Effects on Human Health. Foods 2017;6:E92. doi:10.3390/foods6100092 
18. Kidd PM. Bioavailability and activity of phytosome complexes from botanical polyphenols: the silymarin, curcumin, green tea, and grape seed extracts. Altern Med Rev 2009;14:226-246.

19. Qureshi K, Abrams GA. Metabolic liver disease of obesity and role of adipose tissue in the pathogenesis of nonalcoholic fatty liver disease. World J Gastroenterol 2007;13:3540. doi:10.3748/wjg.v13.i26.3540

20. Li L, Liu DW, Yan HY, Wang ZY, Zhao SH, Wang B. Obesity is an independent risk factor for non-alcoholic fatty liver disease: evidence from a meta-analysis of 21 cohort studies. Obes Rev 2016;17:510-519. doi:10.1111/obr.12407

21. Fabbrini E, Sullivan S, Klein S. Obesity and nonalcoholic fatty liver disease: biochemical, metabolic, and clinical implications. Hepatology 2010;51:679-689. doi:10.1002/hep.23280

22. Di Pierro F, Bressan A, Ranaldi D, Rapacioli G, Giacomelli L, Bertuccioli A. Potential role of bioavailable curcumin in weight loss and omental adipose tissue decrease: preliminary data of a randomized, controlled trial in overweight people with metabolic syndrome. Preliminary study. Eur Rev Med Pharmacol Sci 2015;19:4195-4202.

23. Rupasinghe HV, Sekhon-Loodu S, Mantso T, Panayiotidis MI. Phytochemicals in regulating fatty acid $\beta$-oxidation: Potential underlying mechanisms and their involvement in obesity and weight loss. Pharmacol Ther 2016;165:153-163. doi:10.1016/j. pharmthera.2016.06.005

24. Jeon SM. Regulation and function of AMPK in physiology and diseases. Exp Mol Med 2016;48:e245. doi:10.1038/emm.2016.81

25. Ejaz A, Wu D, Kwan P, Meydani M. Curcumin inhibits adipogenesis in 3T3-L1 adipocytes and angiogenesis and obesity in C57/BL mice. J Nutr 2009;139:919-925. doi:10.3945/jn.108.100966

26. Kang C, Kim E. Synergistic effect of curcumin and insulin on muscle cell glucose metabolism. Food Chem Toxicol 2010;48:2366-2373. doi:10.1016/j.fct.2010.05.073

27. Asai A, Miyazawa T. Dietary curcuminoids prevent high-fat dietinduced lipid accumulation in rat liver and epididymal adipose tissue. J Nutr 2001;131:2932-2935. doi:10.1093/jn/131.11.2932

28. Sahebkar A. A systematic review and meta-analysis of randomized controlled trials investigating the effects of curcumin on blood lipid levels. Clin Nutr 2014;33:406-414. doi:10.1016/j.clnu.2013.09.012

29. Shao W, Yu Z, Chiang Y, et al. Curcumin prevents high fat diet induced insulin resistance and obesity via attenuating lipogenesis in liver and inflammatory pathway in adipocytes. PLoS One 2012;7:e28784 doi:10.1371/journal.pone.0028784

30. Mansour-Ghanaei F, Pourmasoumi M, Hadi A, Joukar F. Efficacy of curcumin/turmeric on liver enzymes in patients with non-alcoholic fatty liver disease: Asystematic review of randomized controlled trials. Integr Med Res 2019;8:57-61. doi:10.1016/j.imr.2018.07.004

31. Kumashiro N, Erion DM, Zhang D, et al. Cellular mechanism of insulin resistance in nonalcoholic fatty liver disease. Proc Natl Acad Sci U S A 2011;108:16381-16385. doi:10.1073/pnas.1113359108

32. Shehzad A, Ha T, Subhan F, Lee YS. New mechanisms and the anti-inflammatory role of curcumin in obesity and obesity-related metabolic diseases. Eur J Nutr 2011;50:151-161. doi:10.1007/s00394011-0188-1

33. Maithilikarpagaselvi N, Sridhar MG, Swaminathan RP, Zachariah B. Curcumin prevents inflammatory response, oxidative stress and insulin resistance in high fructose fed male Wistar rats: Potential role of serine kinases. Chem Biol Interact 2016;244:187-194. doi:10.1016/j. cbi.2015.12.012

34. Vayghan HJ, Esfanjani AT, Ebrahimi Mameghani M, et al. Sex differences in serum leptin and adiponectin levels in apparently healthy iranian adults. Intl Res J Appl Basic Sci 2013;4:3099-3103.

35. Navekar R, Rafraf M, Ghaffari A, Asghari-Jafarabadi M, Khoshbaten M. Turmeric Supplementation Improves Serum Glucose Indices and Leptin Levels in Patients with Nonalcoholic Fatty Liver Diseases. J Am Coll Nutr 2017;36:261-267. doi:10.1080/07315724.2016.1267597

36. Fisher CD, Lickteig AJ, Augustine LM, et al. Experimental non-alcoholic fatty liver disease results in decreased hepatic uptake transporter expression and function in rats. Eur J Pharmacol 2009;613:119-127. doi:10.1016/j.ejphar.2009.04.002

37. Chuengsamarn S, Rattanamongkolgul S, Luechapudiporn R, Phisalaphong C, Jirawatnotai S. Curcumin extract for prevention of type 2 diabetes. Diabetes Care 2012;35:2121-2127. doi:10.2337/dc12-0116

38. Panahi Y, Hosseini MS, Khalili N, et al. Effects of supplementation with curcumin on serum adipokine concentrations: a randomized controlled trial. Nutrition 2016;32:1116-1122. doi:10.1016/j.nut.2016.03.018

39. Weisberg SP, Leibel R, Tortoriello DV. Dietary curcumin significantly improves obesity-associated inflammation and diabetes in mouse models of diabesity. Endocrinology 2008;149:3549-3558. doi:10.1210/ en.2008-0262 